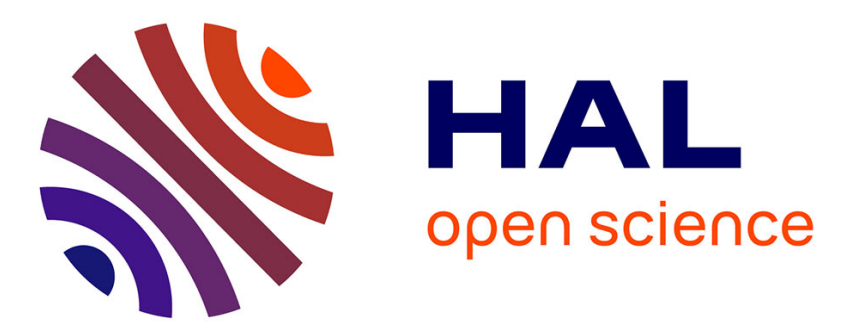

\title{
Etudes génétiques menées en Amazonie: une collaboration entre la Guyane française et le Brésil.
}

Bernd Degen, Milton Kanashiro, Henri Caron, Antoine Kremer, Ian Samuel Thompson

\section{- To cite this version:}

Bernd Degen, Milton Kanashiro, Henri Caron, Antoine Kremer, Ian Samuel Thompson. Etudes génétiques menées en Amazonie: une collaboration entre la Guyane française et le Brésil.. Revue forestière française, 2003, 55 (sp), pp.147-157. 10.4267/2042/5768 . hal-03449394

\section{HAL Id: hal-03449394 \\ https://hal.science/hal-03449394}

Submitted on 25 Nov 2021

HAL is a multi-disciplinary open access archive for the deposit and dissemination of scientific research documents, whether they are published or not. The documents may come from teaching and research institutions in France or abroad, or from public or private research centers.
L'archive ouverte pluridisciplinaire HAL, est destinée au dépôt et à la diffusion de documents scientifiques de niveau recherche, publiés ou non, émanant des établissements d'enseignement et de recherche français ou étrangers, des laboratoires publics ou privés. 


\title{
Études génétiques menées en AmAZonie : UNE COLLABORATION ENTRE LA GUYANE FRANÇAISE ET LE BRÉSIL
}

\author{
Bernd Degen - Milton Kanashiro - Henri Caron \\ Antoine Kremer - IAN Samuel Thompson
}

\section{CONTEXTE}

Bien que les forêts humides de basse altitude ne recouvrent que 6 à $7 \%$ de la superficie des terres de la planète, elles abritent plus de $50 \%$ (jusqu'à $90 \%$, selon certaines estimations) de l'ensemble des espèces vivantes (Linsenmair, 1997). La forêt amazonienne est reconnue comme étant l'un des écosystèmes forestiers présentant la diversité biologique la plus importante au monde. Elle recouvre, rien qu'au Brésil, 3,5 millions de $\mathrm{km}^{2}$. En dépit de la pression internationale visant à réduire la vitesse de déforestation et l'abattage sélectif, en 1996-97, 2500 scieries fonctionnaient encore et consommaient l'équivalent de 27,8 millions de $\mathrm{m}^{3}$ (grumes pour le sciage) de bois tropical (Nepstad et al., 1999).

La situation en Guyane française est très différente de celle du Brésil. La pression anthropique sur la forêt reste marginale. La densité démographique est très faible (160 000 à 200000 habitants). Plus de $90 \%$ de sa superficie est encore recouverte de forêt tropicale vierge (7,5 millions ha). La récolte annuelle est de $65000 \mathrm{~m}^{3}$. En 1999, seuls $8000 \mathrm{~m}^{3}$ de bois ont été exportés. Un massif de 500000 ha est géré par l'ONF. Différentes zones y ont été établies par l'ONF et classées prioritairement pour l'exploitation ou pour la conservation. De plus, il existe un projet de création d'un parc national protégé portant sur une superficie de 1,5 million ha de forêt située dans la partie méridionale de la Guyane française.

Les arbres, comparés aux autres organismes, possèdent un niveau de diversité génétique très élevé (Hamrick et Godt, 1990). Cela s'explique partiellement par les traits d'histoire de vie propres aux arbres (taille de population importante, taux élevé de reproduction allogame, et dispersion de pollen sur grande distance). De plus, ce sont des organismes sans possibilité de déplacement, longévifs et confrontés à des aléas climatiques. La diversité génétique leur est donc en quelque sorte nécessaire pour s'adapter à des conditions environnementales aussi hétérogènes. Afin de préserver la stabilité des écosystèmes forestiers, la conservation des ressources génétiques forestières est un élément important qui doit être pris en compte dans la gestion durable de la forêt amazonienne.

La ressemblance entre les forêts de la Guyane française et du Brésil offre la possibilité de mener conjointement des recherches sur les écosystèmes forestiers peu anthropisés (en Guyane) et ceux soumis à une forte exploitation des ressources (Amazonie brésilienne). Ces comparaisons permettent tout particulièrement, en plus du partage des méthodologies, de mesurer l'impact humain sur la biodiversité, la régénération et la croissance forestières. Grâce aux recherches menées en colla- 
boration entre les deux pays, on sera en mesure d'établir les fondements scientifiques solides qui permettront de formuler des recommandations en matière de gestion durable des forêts.

\section{COLLABORATION EN MATIÈRE DE RECHERCHES GÉNÉTIQUES}

Le début de la coopération entre Silvolab-Guyane et les différents organismes de recherche brésiliens remonte à 1998 (figure 1, ci-dessous). Les différents partenaires brésiliens dans le projet de

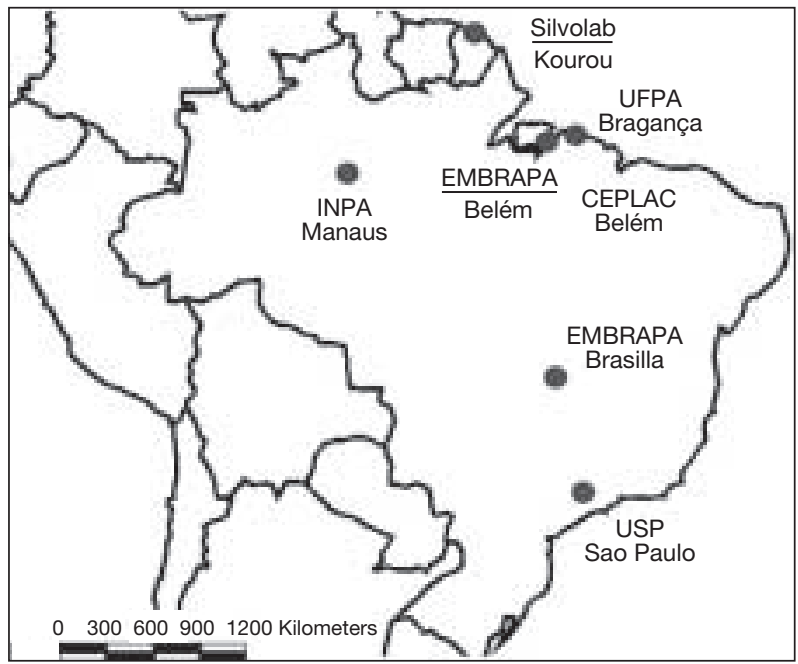
recherches en génétique forestière sont : Empresa Brasileira de Pesquisa Agropecuaria (EMBRAPA), Instituto Nacional de Pesquisas da Amazônia (INPA), Universidade Federal do Pará (UFPA), Universidade Federal do Rio de Janeiro (UFRI), Escola Superior de Agricultura Luiz de Queiroz, da Universidade de São Paulo (ESALQ/USP).

Cette collaboration est principalement issue d'initiatives menées dans le cadre du projet "Dendrogene". Ce dernier, conduit par la station de recherche d'Amazonie orientale d'Embrapa, à Belém (État de Pará), se caractérise par une approche pluridisciplinaire à laquelle collaborent plusieurs instituts ${ }^{(1)}$. La DFID (Department for International Development - Direction pour le Développement international) du Royaume-Uni finance ce projet (période 2000-2004) dans le cadre d'un accord bilatéral entre le Royaume-Uni et le Brésil. Le projet comprend trois objectifs principaux :

- développer et appliquer des modèles de simulation ainsi que d'autres logiciels permettant de prévoir l'impact potentiel de l'exploitation forestière sur la diversité génétique et la démographie des populations d'essences tropicales;

- étudier le niveau et la structure de la diversité génétique d'un ensemble d'essences tropicales ;

- comparer l'évolution et la dynamique de la diversité génétique dans les forêts exploitées et non exploitées.

Le but ultime de ces études est de formuler des recommandations permettant une gestion forestière plus durable, capable de conserver les ressources génétiques des forêts. D'autres aspects de cette collaboration sont financés par le projet INCO “Geneotropico" de l'Union européenne(2) ainsi que par le Fonds de Coopération régionale (FCR) de la Région Guyane.

Sont également prévus dans le cadre des recherches génétiques menées au titre de cette coopération : le développement d'un ensemble de progiciels interactifs ainsi que leur approvisionne-

(1) Cf. http://www.cpatu.embrapa.br/dendro/index.htm

(2) Cf. http://www.edinburgh.ceh.ac.uk/geneo/ 
ment en données, et des études expérimentales comparatives entre les sites de Paracou, en Guyane française, et de Tapajós, au Brésil sur la structure et l'évolution de la diversité génétique de plusieurs espèces. Cet article donne un bref aperçu du travail expérimental et des outils informatiques développés et utilisés dans le cadre de ces projets de coopération.

\section{TRAVAIL EXPÉRIMENTAL}

\section{Site de Paracou $\left.{ }^{3}\right)$}

Paracou est le principal site expérimental pour les études génétiques en Guyane française. II est classé forêt humide de basse altitude sur sol ferrallitique. La pluviosité moyenne annuelle dans cette zone est de $3076 \mathrm{~mm}$ avec des précipitations maximales entre avril et juin et en décembre. Il y a deux saisons sèches - une plus courte en mars et une plus longue qui dure d'août à novembre.

Jusqu'ici les études génétiques ont porté exclusivement sur 8 espèces test dont les traits de vie sont différents (par exemple, modes de dispersion du pollen et des graines, phénologie, type sexuel, etc.) : Carapa procera (Meliaceae), Chrysophyllum sanguinolentum (Sapotaceae), Dicorynia guianensis (Caesalpiniaceae), Eperua grandiflora (Caesalpiniaceae), Moronobea coccinea (Clusiaceae), Symphonia globulifera (Clusiaceae), Virola michelii (Myristicaceae) et Vouacapoua americana (Caesalpiniaceae).

L'utilisation de marqueurs moléculaires a permis de mesurer le niveau de diversité génétique et de vérifier la corrélation avec les différents traits de vie. Le tableau I (ci-dessous) récapitule les résultats de ces études. Nous avons pu observer jusqu'ici que la diversité génétique des essences dont la répartition géographique est la plus large, telles Symphonia globulifera ou Carapa procera, est supérieure à celle des espèces endémiques ou celles dont la répartition géographique est

TABLEAU I Niveau de diversité génétique mesuré par l'hétérozygotie ( $\mathrm{Hp}, \mathrm{He})$ :

probabilité que deux allèles (variants génétiques) soient différents, chez les individus de 8 essences à Paracou selon 4 marqueurs moléculaires différents (RAPD, AFLP, ADN chloroplastique, microsatellites ${ }^{(*)}$ )

Données provenant de Caron (2000), Degen et al. (soumis), Dutech et al. (2000), Dutech et al. (2002) ainsi que Latouche-Hallé et al. (2003)

\begin{tabular}{|c|c|c|c|c|}
\hline Espèces & $\begin{array}{c}\text { RAPD } \\
\mathrm{H}_{\mathrm{p}}\end{array}$ & $\begin{array}{c}\text { AFLP } \\
\mathrm{H}_{\mathrm{p}}\end{array}$ & $\begin{array}{c}\text { ADNcp } \\
\mathrm{H}_{\mathrm{e}}\end{array}$ & $\begin{array}{c}\text { Microsatellites } \\
\mathrm{H}_{\mathrm{e}}\end{array}$ \\
\hline Vouacapoua americana & 0,17 & 0,09 & 0,09 & 0,48 \\
\hline Moronobea coccinea & 0,22 & & 0,00 & \\
\hline Dicorynia guianensis & 0,26 & 0,15 & 0,46 & 0,62 \\
\hline Eperua grandiflora & 0,28 & 0,16 & 0,30 & \\
\hline Virola michelii & 0,32 & 0,21 & 0,58 & \\
\hline Chrysophyllum sanguinolentum & 0,35 & 0,14 & 0,28 & \\
\hline Carapa procera & 0,39 & & & \\
\hline Symphonia globulifera & 0,39 & & 0,77 & 0,80 \\
\hline
\end{tabular}

$\left(^{\star}\right)$ Voir définitions dans la note de bas de page (4) p. 150.

(3) Cf. article de J. Weigel et al. dans ce numéro. 
plus limitée (notamment Vouacapoua americana). Il semblerait également que les espèces dont les graines sont dispersées par les oiseaux, les singes et les chauves-souris présentent un niveau de diversité plus élevé. Dans le cadre du projet "Geneotropico", la gamme d'espèces étudiées a été étendue à 41 espèces, afin de mieux comprendre les relations entre traits d'histoire de vie et diversité.

D'autres études menées à Paracou portent sur l'analyse de la structure génétique spatiale (Boutte, 1999 ; Degen et al., 2001a, b ; Degen et al., soumis ; Doligez et Joly, 1997 ; Dutech et al., 2002 ; Latouche-Hallé et al., 2003). Le principal objet de ces études était de savoir si la distribution spatiale des allèles ou génotypes est aléatoire ou, au contraire, s’il y a agrégation d'allèles semblables et des génotypes associés (autocorrélation spatiale). Étant donné la faible densité des peuplements, la pression anthropique relativement limitée et une dispersion restreinte des graines, nous nous attendions a priori à une structure spatiale plus forte dans le cas des espèces tropicales que pour les espèces tempérées. Nous avons supposé que, dans un milieu exploité, les espèces dont les allèles ou génotypes connaissent une distribution non aléatoire agrégée sont plus sensibles à la perte de diversité génétique du fait des abattages. Les résultats (tableau II, ci-dessous) ont montré que :

- la sensibilité de détection de la structure génétique spatiale n'est pas la même pour tous les marqueurs génétiques ${ }^{(4)}$. Les allozymes sont insensibles, contrairement aux microsatellites et à l'ADN chloroplastique. Ces différences sont liées à la quantité de variation génétique détectée

TABLEAU II Structure génétique spatiale d'arbres adultes à Paracou pour différents types de marqueurs

Les distances indiquent l'expansion au sein de laquelle il y a une structure familiale:

la similitude des marqueurs, entre les arbres, est significativement beaucoup plus forte que pour la moyenne des populations.

ns = aucune structure spatiale significative trouvée.

Données issues de Boutte (1999), Degen et al. (2001a et b), Degen et al. (soumis), Doligez et al. (1997), Dutech et al. (2002), Latouche-Hallé et al. (2003)

\begin{tabular}{|c|c|c|c|c|}
\hline Espèces & RAPD & ADNcp & Allozymes & Microsatellites \\
\hline Vouacapoua americana & $150 \mathrm{~m}$ & $600 \mathrm{~m}$ & & $30 \mathrm{~m}$ \\
\hline Moronobea coccinea & $300 \mathrm{~m}$ & & & \\
\hline Dicorynia guianensis & ns & $100 \mathrm{~m}$ & $60 \mathrm{~m}$ & $100 \mathrm{~m}$ \\
\hline Eperua grandiflora & ns & & ns & \\
\hline Virola michelii & $150 \mathrm{~m}$ & $150 \mathrm{~m}$ & ns & \\
\hline Chrysophyllum sanguinolentum & ns & & ns & \\
\hline Carapa procera & $150 \mathrm{~m}$ & & ns & \\
\hline Symphonia globulifera & ns & $120 \mathrm{~m}$ & & $150 \mathrm{~m}$ \\
\hline
\end{tabular}

(4) Les termes RAPD, AFLP, Microsatellite, Allozyme désignent différentes techniques courantes de caractérisation de la variation génétique.

Les trois premières, plus récentes, observent directement le génome (marquage moléculaire) en amplifiant des fragments d’ADN aussi polymorphes que possible. RAPD signifie "Randomly Amplified Polymorphic DNA". AFLP signifie "Amplified Fragment Length Polymorphism". La technique AFLP est plus sensible que la technique RAPD. Les Microsatellites sont constitués de très petites séquences de nucléotides, répétées et distribuées dans le génome; ils sont des marqueurs extrêmement polymorphes et donc performants.

La dernière méthode, Allozyme, consiste à observer les différentes formes d'un enzyme par des techniques d'électrophorèse. Ceci permet de visualiser également la variation génétique car les différents allèles (mutations) d'un même gène contrôlent directement la synthèse de ces formes multiples, appelées allozymes. Un allèle est une des différentes formes alternatives d'un gène occupant le même locus sur un chromosome particulier. 
par chaque type de marqueur et au mode d'hérédité des marqueurs. Les chloroplastes sont généralement transmis de manière exclusivement maternelle chez la plupart des angiospermes. Par conséquent, seule la dispersion des graines détermine la structure spatiale des variants d'ADN chloroplastique.

- par rapport aux espèces tempérées, les structures génétiques spatiales s'étendent sur des distances plus grandes (pouvant aller jusqu'à $600 \mathrm{~m}$, ce qui correspond à un facteur 10 par rapport aux espèces tempérées). Un flux de gènes asymétrique dû à un flux de pollen étendu et à une dispersion limitée des graines conduit à une autocorrélation spatiale plus large et plus forte pour les marqueurs ADNcp.

Une troisième série d'études menées à Paracou vise à analyser la dynamique de la diversité génétique. Le flux de gènes par dispersion de pollen et de graines est un élément important de la dynamique spatio-temporelle de la diversité génétique. Le flux de gènes a été étudié pour Dicorynia guianensis (Caron et al., 1998 ; Latouche-Hallé et al., 2003). Pour d'autres espèces telles que Symphonia globulifera, Vouacapoua americana et Sextonia rubra, l'analyse est en cours. L'étude de la dispersion du pollen est faite par recherche de paternité en utilisant des empreintes génétiques. Ces empreintes relevées sur les semis de la régénération sont comparées à celles des arbres adultes permettant de la sorte d'assigner un semis à un parent. Comme la position spatiale des arbres adultes est connue, on peut donc rétrospectivement reconstruire la migration du pollen. Plus de $95 \%$ des essences tropicales sont pollinisées par l'intermédiaire de la faune et principalement des abeilles (Bawa et al., 1985). La distance maximum de pollinisation pour les abeilles est de l'ordre de quelques centaines de mètres à quelques kilomètres (Roubik, 1999). Par conséquent, il est fondamental d'avoir une taille adéquate pour les parcelles expérimentales abritant tous les géniteurs paternels potentiels dont les données sont cartographiées et le génotype déterminé. À Paracou, c'est le cas pour les superficies jusqu'à 500 x 500 m. La collaboration avec le Brésil permet de mener ce type d'étude à une échelle beaucoup plus grande (voir Tapajós).

\section{Site de Tapajós}

Les études génétiques menées dans le cadre du projet "Dendrogene" sont centralisées au km 83 , en Forêt nationale de Tapajós, à 175 mètres d'altitude, où la pluviosité annuelle est d'environ $2100 \mathrm{~mm}$, avec une saison plus sèche de juillet à septembre. Ces travaux sont menés conjointement dans le cadre du projet "Gestion forestière durable pour la production de bois" avec l'Institut brésilien de l'Environnement et des Ressources naturelles renouvelable (IBAMA) et l'Organisation internationale des Bois tropicaux (OIBT/ITTO).

Sept espèces d'arbres "test”, qui occupent différentes niches écologiques et présentent des stratégies de vie différentes, sont étudiées au sein de populations suffisamment importantes pour permettre d'estimer les flux de gènes (200 à 400 individus adultes). Ces espèces et leurs familles sont les suivantes : Bagassa guianensis (Moraceae), Carapa guianensis (Meliaceae), Dipteryx odorata (Fabaceae), Hymenaea courbaril (Caesalpiniaceae), Jacaranda copaia (Bignoniaceae), Manilkara huberi (Sapotaceae) et Symphonia globulifera (Clusiaceae).

La phase d'inventaire et de suivi des processus écologiques et génétiques a commencé en 2000 en prévision de l'exploitation du bois programmée pour 2003. L'inventaire forestier en vue de l'exploitation a été étendu, sur le site expérimental, afin d'inclure tous les individus appartenant aux espèces test dont le diamètre est égal ou supérieur à $20 \mathrm{~cm}$. Pour une sous-parcelle de 100 ha, l'inventaire a été étendu aux individus dont le diamètre est de $10 \mathrm{~cm}$.

Ces inventaires commerciaux ont été vérifiés par des observations complémentaires. Bien que l'étude porte sur des arbres généralement considérés comme faciles à identifier, on trouve néan- 
moins des erreurs systématiques d'identification sur le terrain pour plus de la moitié des essences test. Dans le cas le plus grave, $16 \%$ des arbres appartenant à une même espèce ont été faussement identifiés.

Le génotype de tous les arbres des 7 espèces est établi à partir d'échantillons de cambium, par amplification des fragments d'ADN et analyse des empreintes génétiques de type microsatellites. Ensuite, ces données sont couplées avec les cartes de l'inventaire de terrain afin de fournir des cartes de répartition pour chaque espèce et de décrire les structures spatiales génétiques. L'analyse génétique à l'aide de marqueurs microsatellites des graines récoltées sur "arbres pièges" sélectionnés permet de retracer la dispersion du pollen. La collecte de graines sur arbres pièges et l'analyse des génotypes avant et après abattage devraient fournir des informations détaillées quant à l'impact de l'exploitation (notamment du fait de la modification de la densité des arbres adultes et de leur distribution spatiale) sur le flux de gènes, les taux d'autofécondation et les modifications dans la diversité génétique des génotypes des plants issus de graines après une exploitation. De bonnes estimations de la variation du flux de gènes doivent pouvoir être obtenues en récoltant les graines sur 30 arbres pièges chaque année, tandis que la récolte d'au moins 30 graines sur chaque arbre fournira une mesure de la variabilité intra-individuelle du mouvement du pollen.

La phénologie constitue un aspect important du système de reproduction des arbres car le mode de floraison peut agir sur le flux de gènes. La circulation de pollen entre arbres ne peut se faire qu'entre individus fleurissant en même temps. L'échelonnement de la floraison peut ainsi limiter ou même empêcher le flux de gènes. Des relevés détaillés de la phénologie de tous les arbres pièges et des arbres témoins sont effectués. Pour ce faire, il a fallu dégager $45 \mathrm{~km}$ de layons afin d'accéder aux arbres objets de l'étude qui sont très éparpillés. D'autres recherches sur les pollinisateurs et sur la répartition des plants issus de graines et des arbres juvéniles sont également en cours.

Les bases de données sont reliées automatiquement à un système d'information géographique (SIG) pour générer des informations spatiales aisément interprétables et constamment mises à jour par l'EMBRAPA.

\section{Progiciels}

\section{- Modèle de simulation ECO-GENE}

La dynamique spatio-temporelle des structures génétiques des populations d'arbres résulte de divers processus : le flux de gènes, par le pollen puis les graines, d'une génération de plantes à une autre ; le système d'accouplement par lequel les individus de sexe opposé sont croisés pour produire des descendants (autofécondation, organisation des couples, nombre d'accouplements simultanés...) et qui détermine la structure génétique et le potentiel d’évolution des populations naturelles ; la sélection, naturelle ou artificielle, qui modifie la structure génétique d'une population en favorisant la survie et la reproduction d'individus particuliers dont les caractéristiques sont donc héritées, préférentiellement, au cours de générations successives ; la dérive génétique résultant du changement aléatoire dans la fréquence des allèles d'une génération à l'autre. L'exploitation et la fragmentation des forêts peuvent influencer simultanément plusieurs de ces processus. Mesurer l'impact global de ces actions sur la variation génétique des essences tropicales nécessite donc une connaissance de la dynamique de ces processus, dont les effets peuvent être appréhendés par la modélisation.

ECO-GENE a été développé dans cette optique (Degen et al., 1996). Il peut générer des générations chevauchantes ou distinctes selon qu'il y a accouplement entre individus des différentes 
classes d'âge ou non (ce dernier cas correspondant par exemple aux plantes annuelles qui ne se reproduisent qu'une fois). Le modèle peut être appliqué aussi bien à des données virtuelles que réelles et il a déjà été utilisé pour étudier les effets de différentes pratiques sylvicoles et ceux de la pollution atmosphérique sur la structure génétique de populations d'arbres tempérés (Degen et Scholz, 1996 ; Degen et al., 1997 ; Degen et Scholz, 1998 ; Geburek et Mengel, 1998). Depuis 1998, des évolutions ont été apportées à ECO-GENE permettant de l'adapter aux conditions particulières des essences tropicales, notamment par l'ajout de modules pour la dispersion du pollen et des graines et d'un nouveau module (Degen et al., 2002 ; Degen et Roubik, 2004) tenant compte de la phénologie de floraison. Grâce à l'intégration du modèle de croissance Symfor (www.symfor.org), l'application permet désormais une modélisation plus performante des processus de croissance et de l'impact des pratiques sylvicoles (Phillips et al., 2004).

Le modèle ECO-GENE devra être validé à travers une comparaison avec les données réelles recueillies sur une parcelle objet d'études intensives (500 ha) située dans la Forêt nationale de Tapajós et sur le site de Paracou. La génétique, la biologie de reproduction et l'écologie de sept essences produisant du bois d'œuvre sont actuellement étudiées et ces études se poursuivront après les opérations d'abattage de 2003. Notre objectif est d'évaluer l'impact des différentes pratiques de gestion forestière sur la composition génétique des populations d'arbres sur une durée de 50 ou 100 ans. Il s'agit de prévoir le caractère pérenne de différents scénarios de gestion de la forêt afin d'orienter les décisions prises dans le cadre de politiques nationales concernant les ressources forestières.

Parmi les simulations envisagées figurent :

- l'utilisation de critères différents pour sélectionner les arbres exploitables ;

- l'impact des erreurs d'identification des essences ;

- l'impact de différentes répartitions spatiales des arbres abattus ;

- l'impact de différents plans de coupes, intensités d'exploitation à l'intérieur d'une même unité de gestion ;

- l'impact de différentes échelles de gestion, depuis la petite forêt jusqu'au grand massif et au paysage ;

- l'impact de la fragmentation des zones forestières et l'importance de préserver des espaces tampon ripisylves et zones protégées permanentes ;

- l'utilité de différents indicateurs sylvicoles, comme le pourcentage de matériel commercialisable laissé sur pied.

Un exemple de la mise en œuvre d'ECO-GENE est l'évaluation des effets de la récolte du bois et de la fragmentation des forêts sur la diversité génétique de Jacaranda copaia (Degen et al., 2002).

\section{DENDROBASE - Base de données concernant le système génétique des essences tropicales}

DENDROBASE est une base de données qui contient les données génétiques, biologiques et écologiques propres aux essences tropicales (Degen, 1999). Elle comprend également les résultats issus de parcelles expérimentales où ces espèces sont testées. L'objet de cette base de données est de rassembler les connaissances actuelles afin de déterminer, pour certaines espèces ou groupes d'espèces clés, des seuils critiques pour le matériel sur pied, à savoir le matériel minimum qu'il faut laisser en place après récolte. À cet effet, la base de données contient des tableaux récapitulatifs des données concernant le mode de croisement, le niveau de consanguinité, le système de reproduction, les agents de dispersion du pollen et des graines, les paramètres de la variation génétique et de la différentiation génétique entre populations, ainsi que des informations sur l'abondance de l'espèce et son aire de répartition. À défaut de données 
expérimentales pour le modèle de simulation ECO-GENE, DENDROBASE peut servir à produire des données d'entrée pour celui-ci et à définir la gamme pertinente de paramètres du modèle.

L'efficacité de la base de données et la pertinence des analyses sont directement liées à la quantité de connaissances stockées sous forme de tableaux. La meilleure solution pour constituer un tel système est par conséquent d'ouvrir l'accès à la base de données à un réseau. L'accumulation des connaissances produites par différents groupes institutionnels constituerait un important pas en avant dans le cadre de cette coopération entre la Guyane française et le Brésil. Même si, à l'avenir, la modélisation devait montrer ses limites, la base de données à elle seule aura une importance considérable pour la mise au point d'indicateurs et de leurs valeurs seuils pour les besoins de la gestion forestière. Elle constitue également un recueil de connaissances des systèmes génétiques des essences tropicales. Elle devrait contribuer à améliorer la coordination et la définition des priorités en matière de recherche tout en améliorant l'accès aux informations existantes.

\section{TREMA - Utilitaires de cartographie et de gestion des arbres}

TREMA est un logiciel, mis au point par William Hawthorne et Denis Filer (Université de Oxford), actuellement en phase de développement, qui doit servir d'outil de gestion et de cartographie pour les données relatives aux arbres (http://www.trema.co.uk). Il peut être aussi considéré comme un outil capable de gérer quatre types fondamentaux d'information : les données relatives aux espèces, aux caractéristiques spatiales, à celles des individus et du bois. Il a été développé dans le cadre d'une architecture ouverte de façon à ce que des modules spécifiques supplémentaires puissent être élaborés et reliés par interfaçage au système central. Ses applications sont multiples mais ses fonctions essentielles sont la gestion des noms d'espèces, la programmation, le suivi des récoltes d'arbres et la cartographie des informations sur les peuplements forestiers. C'est un dispositif qui permet de mettre les connaissances en matière de génétique et d'écologie reproductive au service de l'aménagement et de la gestion des forêts (Hawthorne et al., 1999).

L'identification des arbres sur le terrain, pratiquée couramment par les forestiers qui utilisent leurs noms communs, doit être faite sur des bases scientifiques car c'est l'espèce qui constitue la base de la biologie de reproduction et qui permet d'accéder aux données techniques telles que les propriétés du bois, ses caractéristiques écologiques et sa valeur économique. Dans cette optique, le système TREMA est interfacé avec l'application BRAHMS, un outil de recherche botanique et de gestion d'herbier. Il faut également établir le lien entre les noms d'espèces et les appellations commerciales du bois pour les besoins de l'industrie. Établir un système de renvois et standardiser les dénominations constituent des étapes compliquées mais primordiales pour améliorer l'utilisation des informations et les compétences en matière de gestion. Le Laboratoire de Produits forestiers de l'IBAMA a mis sa liste des essences brésiliennes à la disposition des responsables de TREMA.

Les dispositifs de gestion de la forêt tropicale sont généralement basés sur des pratiques d'abattage sélectif. Être en mesure de planifier et de contrôler l'intensité et la répartition de cet abattage sélectif à l'échelon de la population d'une essence, dans les limites de ce qui est économiquement viable, constitue un élément indispensable à la gestion forestière durable. Le module de planification de la récolte permet d'appliquer une approche multicritères aux données sur les peuplements afin de déterminer le choix des arbres à abattre ou à maintenir.

Les critères qui jouent sur le choix des arbres relèvent de facteurs tels que les réglementations locales en matière d'abattage, l'état du marché pour les produits de la forêt, de même que les critères économiques et écologiques à court et à long termes. Les données réelles concernant les 
résultats de la récolte, et notamment les arbres effectivement abattus, peuvent être intégrées pour évaluer l'efficacité du mode d'exploitation. On peut établir un rapport entre production de grumes et récolte d'arbres, ce qui permet potentiellement d'évaluer les résultats économiques et suivre la chaîne de traçabilité jusqu'à la transformation. TREMA permet également d'éditer des tableaux de données sur les peuplements et des rapports de récolte pour les besoins de la gestion prévisionnelle.

Le module de planification des récoltes intègre une fonction d'affichage des informations sur carte, ce qui permet de nombreuses applications et notamment l'analyse de la répartition géographique qui peut aider aux choix des arbres, à l'aménagement de chemins d'exploitation, et à la conduite des opérations d'abattage et de mobilisation du bois.

\section{CONCLUSIONS}

La coopération en cours s'est déjà concrétisée par des résultats très positifs. Elle mérite d'être soutenue et renforcée par d'autres institutions de la Guyane et le Brésil. Elle pourrait être étendue au concours d'institutions d'autres pays voisins préoccupés par les mêmes sujets d'intérêt. Le renforcement de la coopération permettrait aussi d'améliorer les transferts d'information entre les différentes initiatives en cours, de satisfaire à des besoins pédagogiques et de limiter le coût de ces recherches.
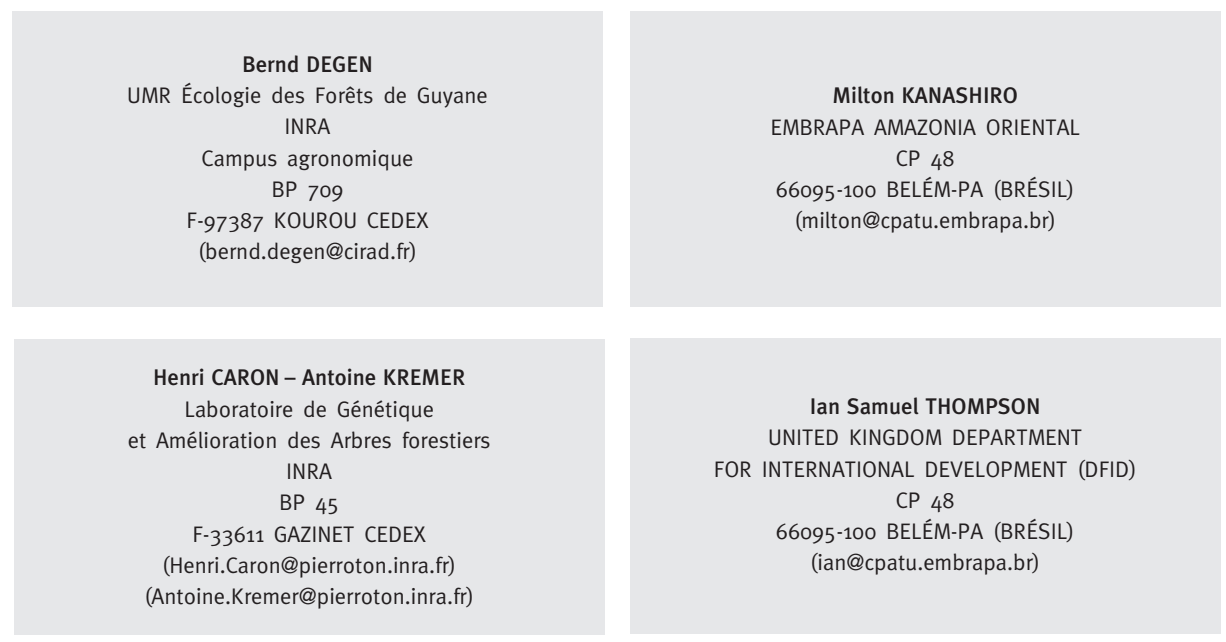


\section{BIBLIOGRAPHIE}

BAWA (K.S.), BULLOCK (S.H.), PERRY (D.R.), COVILLE (R.E.), GRAYUM (M.H.). - Reproductive biology of tropical lowland rain forest trees. II. Pollination system. - American Journal of Botany, vol. 72, 1985, pp. 346-356.

BOUTTE (C.). - Étude du polymorphisme de l'ADN chloroplastique de deux espèces tropicales : Monorobea coccinea L. et Symphonia globulifera L. - Bordeaux-Cestas : INRA-Laboratoire de Génétique et d'Amélioration des Arbres forestiers ; Nancy : Université Henri-Poincaré, 1999. - 20 p.

CARON (H.). - Organisation et dynamique de la diversité génétique de cinq espèces arborées de la forêt guyanaise. - Montpellier : Université de Montpellier, 2000. - 88 p.

CARON (H.), DUTECH (C.), BANDOU (E.). - Variations spatiotemporelles du régime de reproduction de Dicorynia guianensis Amshoff (Caesalpiniaceae) en forêt guyanaise. - Genet. Sel. Evol., vol. 30, 1998, S153$\mathrm{S} 166$.

DEGEN (B.). - Dendrobase : Genetic system of tropical tree species. Users's manual for the data base. 1999. - 29 p. (http://kourou.cirad.fr/genetique/Software/Dendrobase.pdf).

DEGEN (B.), ROUBIK (D.W.), LOVELESS (M.D.). - Impact of selective logging and forest fragmentation on the seed cohorts of an insect-pollinated tropical tree : a simulation study. pp. 104-114. In : Modelling and experimental research on genetic processes in tropical and temperate forests / B. Degen, M. Loveless, A. Kremer Eds. - Belém : EMBRAPA, 2002. - 211 p.

DEGEN (B.), GREGORIUS (H.-R.), SCHOLZ (F.). - ECO-GENE, a model for simulation studies on the spatial and temporal dynamics of genetic structures of tree populations. - Silvae Genetica, vol. 45, 1996, pp. 323329.

DEGEN (B.), CARON (H.), BANDOU (E.), DUTECH (C.), BREYNE (P.), MONTAGOU (M.V.), MAGGIA (L.), KREMER (A.). - Small scale spatial genetic structure of six tropical tree species in French Guiana. pp. 7592. In : Genetic Response of Forest Systems to changing Environmental Conditions / G. Müller-Starck, R. Schubert Eds. - Dordrecht : Kluwer Academic Publisher, 2001 .

DEGEN (B.), CARON (H.), BANDOU (E.), MAGGIA (L.), ChEVALLIER (M.H.), LEVEAU (A.), KREMER (A.). - Finescale spatial genetic structure of eight tropical tree species as analysed by RAPDs. - Heredity, vol. 87 , 2001b, pp. 497-507.

DEGEN (B.), SCHOLZ (F.). - Der Einsatz des Simulationsmodells ÖKO-GEN zur Erarbeitung von Entscheidungshilfen für eine nachhaltige Forstwirtschaft. pp. 284-299. In : Biodiversität und nachhaltige Forstwirtschaft / G. Müller-Starck Ed. . - Landsberg : Ecomed - Verlagsgesellschaft, 1996.

DEGEN (B.), SCHOLZ (F.). - Ecological genetics in forest ecosystems under stress as analysed by the simulation model ECO-GENE. - Chemosphere, vol. 36, 1996, pp. 819-824.

DEGEN (B.), STREIFF (R.), SCHOLZ (F.), KREMER (A.). - Analyzing the effects of regeneration regime on genetic diversity and inbreeding in oak populations by use of the simulation model ECO-GENE. pp. 9-21. In : Diversity and adaptation in oak species / K.C. Steiner Ed. . - Pennstate : College of Agricultural Sciences, University Park, Pennsylvania, 1997.

DEGEN (B.), ROUBIK (D.). - Effects of animal pollination on pollen dispersal, self-pollination and effective population size of tropical trees : a simulation study. - Biotropica, vol. 36, $\mathrm{n}^{\circ}$ 2, 2004.

DEGEN (B.), BANDOU (E.), CARON (G.). - Limited pollen dispersal and biparental inbreeding in Symphonia globulifera in French Guiana. - (soumis pour publication).

DOLIGEZ (A.), JOLY (H.I.). - Genetic diversity and spatial structure within a natural stand of a tropical forest tree species, Carapa procera (Meliaceae), in French Guiana. - Heredity, vol. 79, 1997, pp. 72-82.

DUTECH (C.), SEITER (J.), PETRONELLI (P.), JOLY (H.I.), JARNE (P.). - Evidence of low gene flow in a neotropical clustered tree species in two rainforest stands in French Guiana. - Molecular Ecology, vol. 11, 2000, pp. 725-738.

DUTECH (C.), MAGGIA (L.), JOLY (H.I.). - Chloroplast diversity in Vouacapoua americana (Caesalpiniaceae), a neotropical forest tree. - Molecular Ecology, vol. 9, 2000, pp. 1427-1432.

GEBUREK (T.), MENGEL (M.). - Considerations in view of identity control of forest reproductive material and minimum number of seed trees required by law as exemplarity described in European larch. - Centralblatt für das gesamte Forstwesen, vol. 115, 1998, pp. 211-228.

HAMRICK (J.L.), GODT (M.J.W.). - Allozyme diversity in plant species. pp. 43-63. In : A.H.D. Brown, M.T. Clegg, A. L. Kahler, B.S. Weir Eds. - Sunderland/MA : Sinauer Associates, 1990. 
HAWTHORNE (W.D.), FILER (L.D.), THOMPSON (I.S.). - 0 desenvolvimento e implementação do aplicativo TREMA de mapeamento e do planaejamento de colheita para seleção de arvores na Embrapa Amazônia Oriental. Simpósio Silvivultura na Amazônia Oriental : Contribuições do projeto EMBRAPA/DFID, Resumos Expandidos. 133-137. - 1999. - 303 p.

KANASHIRO (M.), THOMPSON (I.S.), YARED (J.A.G.), LOVELESS (M.D.), COVENTRY (P.), MARTINS-DASILVA (R.C.V.), DEGEN (B.), AMARAL (W.). - Improving conservation values of managed forests : the Dendrogene Project in the Brazilian Amazon. - Unasylva, vol. 53, $\mathrm{n}^{\circ}$ 209, 2002, pp. 25-33.

LATOUCHE-HALlÉ (C.), RAMBOER (A.), BANDOU (E.), CARON (H.), KREMER (A.). - Nuclear and chloroplast genetic structure indicate fine-scale spatial dynamics in a neotropical tree population. - Heredity, vol. 91, 2003, pp. 181-190.

LISENMAIR (K.E.). - Biodiversity and sustainable management of tropical forests. In : Focus : Tropical Forests, edited by Natural Resources and Development, $\mathrm{n}^{\circ} 45 / 46,1997$, pp. 13-27.

NEPSTAD (D.C.), VERÍSSIMO (A.), ALENCAR (A.), NOBRE (C.), LIMA (E.), LEFEBVRE (P.), SCHELESINGER (P.), POTTER (C.), MOUTINHO (P.), MENDONZA (E.), COCHRANE (M.), BROOKS (V.). - Large scale impoverishment of Amazonian forests by logging and fire. - Nature, $\mathrm{n}^{\circ} 398,1999$, pp. 505-508.

PHILLIPS (P.D.), DE AZEVEDO (C.P.), DEGEN (B.), VAN GARDINGEN (P.R.), THOMPSON (I.S.), SILVA (N.M.). An individual-based spatially explicit simulation model for strategic forest management planning in the eastern Amazon. - Ecological Modelling, $\mathrm{n}^{\circ}$ 173, 2004, pp. 335-354.

ROUBIK (D.). - The foraging and potential outcrossing pollination ranges of African honey bees (Apiformes : Apidae ; Apini) in Congo forest. - J. Kans. Entomol. Soc., vol. 72, 1999, pp. 394-401.

ZACHOW (R.). - Os planos de manejo das florestas naturais no Brasil. Curso de Manejo Sustentável. Curitiba-Pr (Brazil) : Embrapa Florestas, 1996.

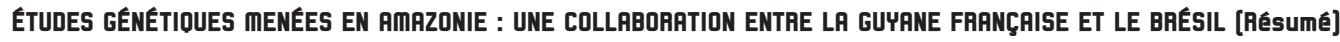

La conservation des ressources génétiques forestières garantit le maintien d'une diversité élevée, donc l'adaptation des espèces forestières tropicales aux conditions écologiques actuelles et futures. Elle nécessite d'élaborer des stratégies appropriées à l'aménagement des forêts et, pour cela, d'appréhender tout d'abord le niveau et la structure de la diversité génétique, de comprendre et prévoir ensuite sa dynamique. C'est dans cet esprit qu'une collaboration a été entreprise entre la Guyane française et le Brésil, malgré les différences existant entre les deux systèmes de gestion. À partir de résultats sur les parcelles expérimentales de Paracou (Guyane) et Tapajós (Brésil), un ensemble intégré d'outils a été élaboré, allant d'une base de données et d'utilitaires cartographiques jusqu'à un modèle de simulation de la dynamique des structures génétiques et à un logiciel de gestion.

\section{GENETIC RESERRCH IN THE AMRZON - A JOINT PROJECT BETUEEN FRENCH GUIANR AND BRAZIL [Abstract]}

Conservation of genetic resources in forests guarantees the preservation of a high degree of diversity thereby ensuring that tropical forest species are able to adapt to present and future ecological conditions. It requires appropriate strategies to be developed for the purposes of forest planning that rely firstly on an appreciation of the level and structure of genetic diversity, and secondly an understanding of its dynamics and the capacity to predict the latter. This is the underlying aim in the joint effort that has been undertaken by French Guiana and Brazil, in spite of the differences between the two management systems. Based on the results obtained in the Paracou (French Guiana) and Tapajos (Brazil) experimental plots, an integrated set of tools has been devised ranging from a data base and cartographic utilities to a model for simulating the dynamics of the genetic structures and a management software package. 